Article

\title{
Catalytic Cracking of Triglyceride-Rich Biomass toward Lower Olefins over a Nano-ZSM-5/SBA-15 Analog Composite
}

\section{Xuan Hoan Vu ${ }^{1, *}$, Sura Nguyen ${ }^{1}$, Tung Thanh Dang ${ }^{1}$, Binh Minh Quoc Phan ${ }^{1}$, \\ Duc Anh Nguyen ${ }^{1}$, Udo Armbruster ${ }^{2}$ and Andreas Martin ${ }^{2, *}$}

1 Vietnam Petroleum Institute, 167 Trung Kinh, Yen Hoa, Cau Giay, Hanoi 10000, Vietnam; E-Mails: suran@pvpro.com.vn (S.N.); tungdt01@vpi.pvn.vn (T.T.D.); binhpmq@vpi.pvn.vn (B.M.Q.P.); duc.a.nguyen@vpi.pvn.vn (D.A.N.)

2 Leibniz-Institut für Katalyse e.V. an der Universität Rostock, Albert-Einstein-Straße 29a, Rostock 18059, Germany; E-Mail: udo.armbruster@catalysis.de

* Authors to whom correspondence should be addressed; E-Mails: hoanvx.ctat@vpi.pvn.vn (X.H.V.); andreas.martin@catalysis.de (A.M.); Tel.: +84-4-3784-3061 (ext. 2092) (X.H.V.); +49-381-1281-246 (A.M.); Fax: +84-4-3629-0107 (X.H.V.); +49-381-1281-51246 (A.M.).

Academic Editor: Keith Hohn

Received: 21 August 2015 / Accepted: 30 September 2015 / Published: 10 October 2015

\begin{abstract}
The catalytic cracking of triglyceride-rich biomass toward $\mathrm{C}_{2}-\mathrm{C}_{4}$ olefins was evaluated over a hierarchically textured nano-ZSM-5/SBA-15 analog composite (ZSC-24) under fluid catalytic cracking (FCC) conditions. The experiments were performed on a fully automated Single-Receiver Short-Contact-Time Microactivity Test unit (SR-SCT-MAT, Grace Davison) at $550{ }^{\circ} \mathrm{C}$ and different catalyst-to-oil mass ratios $\left(0-1.2 \mathrm{~g} \cdot \mathrm{g}^{-1}\right)$. The ZSC-24 catalyst is very effective for transformation of triglycerides to valuable hydrocarbons, particularly lower olefins. The selectivity to $\mathrm{C}_{2}-\mathrm{C}_{4}$ olefins is remarkably high (>90\%) throughout the investigated catalyst-to-oil ratio range. The superior catalytic performance of the ZSC-24 catalyst can be attributed to the combination of its medium acid site amount and improved molecular transport provided by the bimodal pore system, which effectively suppresses the secondary reactions of primarily formed lower olefins.
\end{abstract}

Keywords: lower olefins; cracking of triglyceride; ZSM-5; SBA-15; FCC 


\section{Introduction}

Lower olefins, also known as light olefins (ethene, propene, and butenes), are important feedstock for the production of valuable polymers and chemicals such as polyethylene (PE), polypropylene (PP), methyl tert-butyl ether (MTBE), and ethyl tert-butyl ether (ETBE). The major fraction of produced lower olefins currently stems from petroleum feedstock [1,2], and the increasing demand along with the oil depletion has led to an increased interest in production of those olefins from renewable feedstock such as biomass [3]. In this respect, the processing of triglyceride-rich biomass by fluid catalytic cracking (FCC) units in petroleum refineries represents a promising option for the future to produce renewable liquid fuels and lower olefins $[4,5]$. The utilization of the existing refining infrastructure and configuration for the conversion of triglyceride-rich biomass would require little additional capital investment. However, the yields of gasoline and lower olefins decrease sharply when cracking highly unsaturated triglyceride feedstock over conventional FCC zeolite catalysts (typically ultra-stable zeolite Y (USY)). This is because the large micropore size of zeolite $\mathrm{Y}$ (pore opening size of $0.74 \mathrm{~nm}$; pore intersection size (supercage) of $1.3 \mathrm{~nm}$ ) tends to promote the fast aromatization of unsaturated fatty acids, resulting in a large fraction of heavy aromatic species that are less crackable [6,7].

To avoid such a phenomenon, medium pore size offering zeolite ZSM-5 has been investigated in the cracking of triglycerides [8-10]. With the pore opening size of $0.52-0.56 \mathrm{~nm}$ and pore intersection size of $0.8 \mathrm{~nm}$, ZSM-5 selectively directs the cracking process toward the formation of $\mathrm{C}_{5}-\mathrm{C}_{10}$ hydrocarbons corresponding to the gasoline-boiling range while avoiding the formation of poly-aromatic species. Unfortunately, a high gas yield with low concentrations of light olefins is generally observed $[9,10]$, implying the occurrence of undesired secondary reactions because of the diffusion limitations caused by the relatively small pore size of ZSM-5. Accordingly, the modification of ZSM-5 zeolites to enhance the selectivity to $\mathrm{C}_{2}-\mathrm{C}_{4}$ olefins has been extensively studied in recent years. It has been reported that the shortened diffusion path length and reduced amount of acid sites were favorable for the formation of lower olefins in the cracking of triglyceride-rich biomass [11-13].

In our previous work [14], we have successfully developed a novel nano-ZSM-5-based composite by dispersing nano-ZSM-5 in well-ordered, highly condensed mesoporous SBA-15 analogs via a two-step process. The resulting nano-ZSM-5/SBA-15 analog composites (ZSC) exhibited high hydrothermal stability and improved acidic properties while their accessibility was enhanced by the bimodal pore system. This was clearly proved by gas-phase cracking of model compounds 1,3,5- triisopropylbenzene and cumene in a conventional tube reactor and comparison to H-ZSM-5 and Al-SBA-15 as benchmarks [14]. In the present contribution, we like to report on the application of such ZSC catalysts for the efficient conversion of triglyceride-rich biomass toward lower olefins under FCC conditions. The standard test procedure using the SR-SCT-MAT unit was employed for the catalyst evaluation as it allows simulating an industrial FCC unit more accurately, thereby improving the practical relevance of the results [15]. 


\section{Results and Discussion}

\subsection{Physico-Chemical Properties of the Catalysts}

The catalysts ZSC-24, Al-SBA-15, and commercial H-ZSM-5 were thoroughly characterized by various techniques and model reactions as presented in the earlier work [14]. However, the main catalyst characteristics are summarized in Table 1. In general, Al-SBA-15 possesses the largest external surface (hexagonal mesopores) and the smallest amount of acid sites. Contrarily, commercial H-ZSM-5 shows the least external surface and the highest amount of acid sites, mostly located in micropores. These named properties of ZSC-24, which was prepared from the ZSM-5 precursor solution with $24 \mathrm{~h}$ precrystalization time, are in between, as the previous studies evidenced that this material indeed comprises hexagonal mesoporous domains wherein ZSM-5 nanoparticles are highly dispersed.

Table 1. Physico-chemical properties of ZSC-24, Al-SBA-15, and commercial H-ZSM-5.

\begin{tabular}{|c|c|c|c|c|c|c|}
\hline Catalyst & $\begin{array}{l}\mathrm{SiO}_{2} / \mathrm{Al}_{2} \mathrm{O}_{3}{ }^{\mathrm{a}} \\
(\mathrm{mol} / \mathrm{mol})\end{array}$ & $\begin{array}{c}S_{\text {BET }} \\
\left(\mathrm{m}^{2} \cdot \mathrm{g}^{-1}\right)\end{array}$ & $\begin{array}{l}S_{\text {ext/meso }}{ }^{b} \\
\left(\mathrm{~m}^{2} \cdot \mathrm{g}^{-1}\right)\end{array}$ & $\begin{array}{c}V_{\text {micro }} b \\
\left(\mathrm{~m}^{3} \cdot \mathrm{g}^{-1}\right)\end{array}$ & $\begin{array}{c}V_{t} \\
\left(m^{3} \cdot g^{-1}\right)\end{array}$ & $\begin{array}{l}\text { Acid site amount } \\
\left(\mathrm{mmol} \mathrm{NH}_{3} \cdot \mathrm{g}^{-1}\right)\end{array}$ \\
\hline ZSC-24 & 60 & 361 & 233 & 0.058 & 0.84 & 0.34 \\
\hline Al-SBA-15 & 90 & 446 & 446 & 0 & 0.93 & 0.18 \\
\hline H-ZSM-5 & 22 & 373 & 110 & 0.113 & 0.22 & 1.24 \\
\hline
\end{tabular}

\subsection{Catalyst Evaluation}

Catalytic cracking of triglyceride-rich biomass is generally initiated by thermal decomposition of triglyceride molecules into free fatty acids by means of free radical mechanism. Subsequently, the acid zeolite-based catalyst controls the process and converts free fatty acids into oxygen-containing products (mainly $\mathrm{CO}, \mathrm{CO}_{2}$, and water) and a mixture of hydrocarbons lumped into gaseous hydrocarbon, gasoline, light cycle oil (LCO), and heavy cycle oil (HCO) fractions [6]. It has been reported that the conversion and product selectivity heavily depend on the unsaturation degree of triglycerides under FCC conditions [6,7]. Therefore, the catalytic cracking of triolein comprising predominantly unsaturated triglycerides (technical grade triolein, Santa Cruz Biotechnology) as model feedstock was first carried out over the ZSC-24 catalyst under different cracking severities to study the catalytic behavior and to optimize the cracking conditions. Then the catalytic performance of ZSC-24 relative to that of reference catalysts Al-SBA-15 and H-ZSM-5 was evaluated in the cracking of waste cooking oil (WCO; obtained from a local restaurant) consisting of less unsaturated triglycerides. The effect of the unsaturation level can be assessed by comparing the catalytic results obtained from the cracking of triolein and those obtained from the cracking of WCO. The fatty acid and elemental composition of triolein and WCO are given in Table 2. 
Table 2. Fatty acid and elemental compositions of technical grade triolein and waste cooking oil (WCO).

\begin{tabular}{ccc}
\hline Feedstock & Triolein & WCO \\
\hline Fatty acid composition as wt. \% methyl esters & \\
\hline Dodecanoic acid [C12:0] & - & 1.2 \\
Palmitic acid [C16:0] & 1.1 & 37.2 \\
Stearic acid [C18:0] & 1.9 & 4.9 \\
Oleic acid [C18:1] & 75.6 & 48.8 \\
Linoleic acid [C18:2] & 21.4 & 7.9 \\
\hline Elemental composition, wt. \% & \\
\hline Carbon & 79.4 & 79.4 \\
Hydrogen & 12.0 & 12.6 \\
Oxygen & 8.6 & 7.8 \\
Nitrogen & 0 & 0.2 \\
\hline
\end{tabular}

$[\mathrm{Cx}: y]$ where $x$ is the number of carbon atoms and $y$ is the number of double bonds.

\subsubsection{Catalytic Cracking of Triolein}

The cracking of triolein was carried out over the ZSC-24 catalyst under different severities by varying the catalyst-to-oil (CTO) mass ratio from 0.2 to $1.2 \mathrm{~g} \cdot \mathrm{g}^{-1}$ at $550{ }^{\circ} \mathrm{C}$. In general, the effect of thermal cracking becomes significant at elevated temperatures $\left(>460^{\circ} \mathrm{C}\right)$; thus, the thermal cracking of triolein over an inert material (glass beads) was conducted in a blank test at $550{ }^{\circ} \mathrm{C}$ to reveal the effect of thermal degradation under the investigated conditions. The catalytic results are given in Table 3 and Figure 1.

Table 3. The catalytic performance of ZSC-24 catalyst in the cracking of triolein under different severities by varying the CTO ratio from 0 (glass beads) to $1.2 \mathrm{~g} \cdot \mathrm{g}^{-1}$ at $550{ }^{\circ} \mathrm{C}$.

\begin{tabular}{ccccccc}
\hline & CTO ratio $\left(\mathbf{g} \cdot \mathbf{g}^{-\mathbf{1}}\right.$ ) & $\mathbf{0}$ & $\mathbf{0 . 2}$ & $\mathbf{0 . 4}$ & $\mathbf{0 . 8}$ & $\mathbf{1 . 2}$ \\
\hline & Conversion (wt. \%) & 24.7 & 62.5 & 70.9 & 77.5 & 79.6 \\
\hline & Total gas & 6.4 & 21.0 & 28.0 & 38.9 & 41.0 \\
& Dry gas & 1.3 & 2.1 & 2.3 & 3.0 & 4.0 \\
& LPG & 1.7 & 13.1 & 18.0 & 28.9 & 33.0 \\
Product & $\mathrm{C}_{2}-\mathrm{C}_{4}$ olefins & 1.9 & 13.5 & 18.2 & 29.4 & 33.5 \\
yields (wt. & $\mathrm{CO}, \mathrm{CO}_{2}$ & 3.5 & 5.8 & 7.6 & 7.1 & 4.1 \\
$\%$ \%) & $\mathrm{C}_{5+}$ Gasoline & 17.0 & 34.9 & 35.2 & 29.7 & 28.8 \\
& LCO & 29.6 & 22.4 & 18.7 & 14.4 & 12.1 \\
& HCO & 45.7 & 15.1 & 10.4 & 8.1 & 8.3 \\
& Coke & 0.1 & 1.0 & 1.6 & 2.4 & 2.3 \\
& Water & 1.1 & 5.6 & 6.1 & 6.5 & 7.5 \\
\hline \multicolumn{2}{c}{ Selectivity to $\mathrm{C}_{2}-\mathrm{C}_{4}$ olefins $(\%)^{\text {a }}$} & 67.7 & 90.9 & 91.7 & 93.7 & 92.0 \\
\hline
\end{tabular}

wt. \% on a feed basis. ${ }^{\text {a }}$ fraction of $\mathrm{C}_{2}-\mathrm{C}_{4}$ olefins per total $\mathrm{C}_{2}-\mathrm{C}_{4}$ hydrocarbons.

From Table 3, it can be seen that the thermal cracking (blank test) of triolein occurs noticeably at $550{ }^{\circ} \mathrm{C}$, giving a conversion of $24.7 \mathrm{wt}$. \%. However, the low fraction of short-chain products suggests that most of the heavy oxygenated compounds formed by the decomposition of triglycerides were not converted in the absence of catalyst, which is in line with the previous reports $[6,8]$. In the presence of the 
ZSC-24 catalyst, the yields of light products such as gas and gasoline rise dramatically at the expense of heavy fractions such as HCO and LCO. This indicates that the heavy oxygenated compounds have been effectively converted over ZSC-24 into valuable hydrocarbons, particularly gasoline and lower olefins.

As shown in Table 3, the CTO ratio increase from 0.2 to $1.2 \mathrm{~g} \cdot \mathrm{g}^{-1}$ steadily enhances the conversion and the $\mathrm{C}_{2}-\mathrm{C}_{4}$ olefin yield from 62.5 and 13.5 wt. \% to 79.6 and 33.5 wt. \%, respectively. This corresponds to increases by factors of approximately 1.27 and 2.48 and can be explained by secondary cracking reactions of intermediate fatty acids being mainly activated by the catalysts' acid sites $[8,10,16]$ and leading to the production of desirable hydrocarbons. Hence, increasing the CTO ratio provided more active acid sites in the reaction system; therefore, more gasoline and lower olefins were produced.

Unlike the conversion and $\mathrm{C}_{2}-\mathrm{C}_{4}$ olefin yield, the gasoline yield apparently goes through a maximum when varying the $\mathrm{CTO}$ ratio at $550{ }^{\circ} \mathrm{C}$ (Table 3). In fact, the gasoline yield shows a little gain from 34.9 to 35.2 wt. \% with the CTO ratio increase from 0.2 to $0.4 \mathrm{~g} \cdot \mathrm{g}^{-1}$ and then drops sharply to 29.7 and 28.8 wt. \% at the CTO ratios of 0.8 and $1.2 \mathrm{~g} \cdot \mathrm{g}^{-1}$, respectively. This implies that the over-cracking of gasoline hydrocarbons has been promoted as the CTO ratio exceeded $0.4 \mathrm{~g} \cdot \mathrm{g}^{-1}$, giving rise to a substantial reduction in the gasoline yield [17]. Thus, the CTO ratio should not be higher than $0.4 \mathrm{~g} \cdot \mathrm{g}^{-1}$ under the investigated conditions as the production of gasoline and light olefins are targeted.

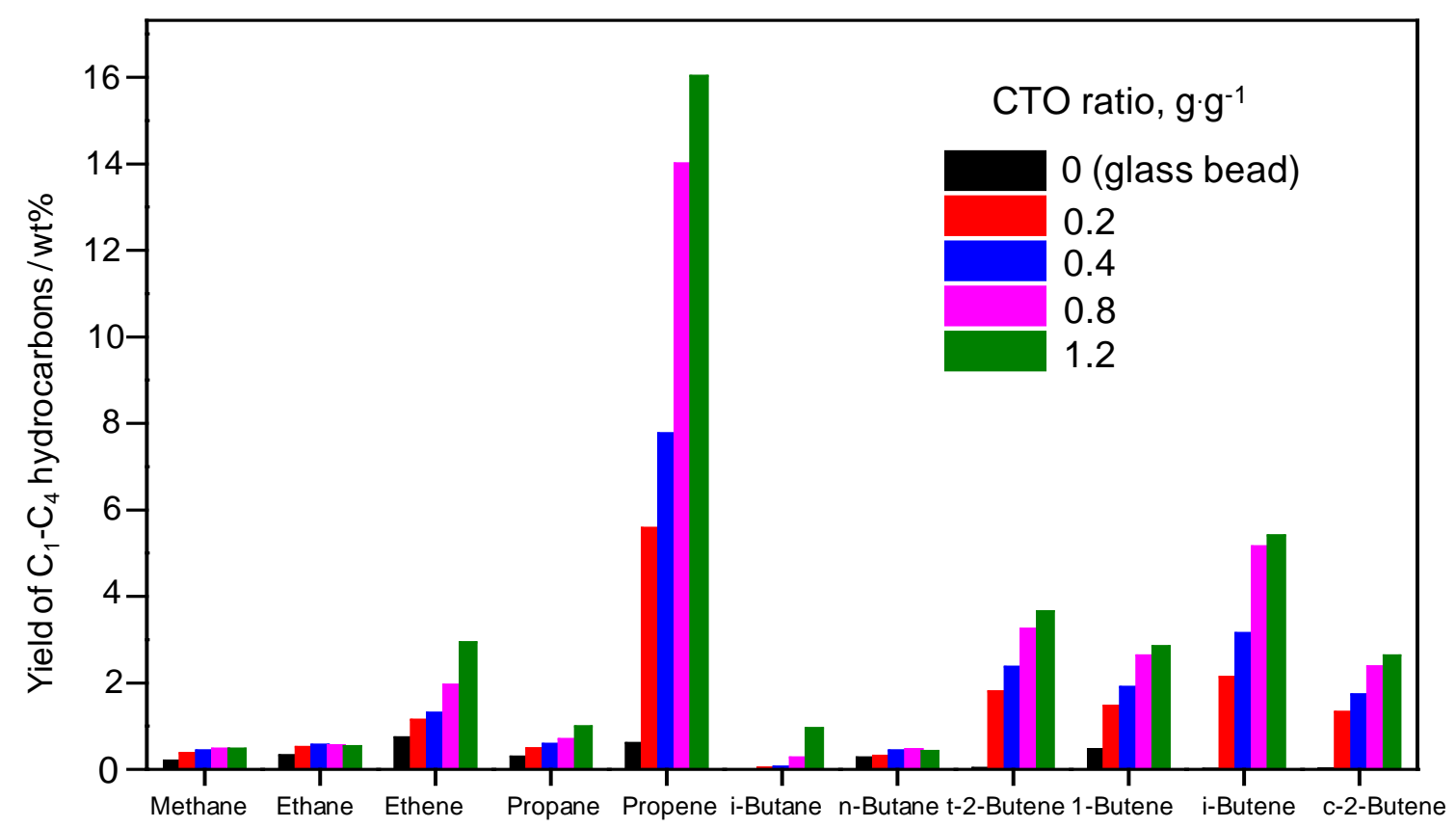

Figure 1. Yields of $\mathrm{C}_{1}-\mathrm{C}_{4}$ hydrocarbons in the cracking of triolein under different severities by varying the CTO ratio from 0 (glass beads) to $1.2 \mathrm{~g} \cdot \mathrm{g}^{-1}$ at $550{ }^{\circ} \mathrm{C}$.

Since the main target of this work is to enhance the selectivity to lower olefins over the ZSC-24 catalyst, the yield of gaseous hydrocarbons was analyzed in detail and the $\mathrm{C}_{2}-\mathrm{C}_{4}$ olefin selectivity was estimated (Figure 1 and Table 3). Interestingly, the selectivity to $\mathrm{C}_{2}-\mathrm{C}_{4}$ olefins is remarkably high, independent of the cracking severity, signifying that the ZSC-24 catalyst is a highly selective catalyst for the conversion of triglyceride-rich biomass to lower olefins. Botas et al. [12] studied the cracking of rapeseed oil over nano-ZSM-5 using a fixed bed reactor. It was found that the shortened diffusion path lengths along with the reduced acid site amount prevented the further transformation of primary 
$\mathrm{C}_{2}-\mathrm{C}_{4}$ olefins, thereby increasing the yield and selectivity to lower olefins. Considering this, it is reasonable that the superior selectivity to $\mathrm{C}_{2}-\mathrm{C}_{4}$ olefins in the cracking of triolein over ZSC-24 originates from the combination of the catalyst's medium acid site amount and improved diffusion characteristics provided by the bimodal pore system.

\subsubsection{Catalytic Cracking of WCO}

The performance of ZSC-24 compared to the reference catalysts Al-SBA-15 and H-ZSM-5 was evaluated in the catalytic cracking of real feedstock WCO under optimized cracking conditions $\left(T=550{ }^{\circ} \mathrm{C}\right.$ and CTO ratio $\left.=0.4 \mathrm{~g} \cdot \mathrm{g}^{-1}\right)$. First, the effect of thermal cracking of WCO was checked in a blank test at $550{ }^{\circ} \mathrm{C}$ using glass beads as inert material.

Table 4 gives the overall data on the catalytic performance of the different catalysts. One can see that there is a significant difference in the conversion of WCO. Commercial H-ZSM-5 looks highly active, showing an almost complete conversion of 91.5 wt. \%, followed by ZSC-24 (70.6 wt. \%) and Al-SBA-15 (58.3 wt. \%). Without the presence of the catalyst, the thermal degradation of WCO yields the least conversion of 26.4 wt. \%. A good correlation of the conversion and the acid site amount can be observed (Tables 1 and 4). This further supports the important role of acid sites in the conversion of intermediate fatty acids into lighter and more valuable hydrocarbons $[8,10]$.

Table 4. Performance of ZSC-24 and the reference catalysts Al-SBA-15 and H-ZSM-5 in the cracking of $\mathrm{WCO}$ at $550{ }^{\circ} \mathrm{C}$ and a CTO ratio of $0.4 \mathrm{~g} \cdot \mathrm{g}^{-1}$.

\begin{tabular}{|c|c|c|c|c|c|}
\hline \multicolumn{2}{|c|}{ Catalyst } & Glass beads & ZSC-24 & H-ZSM-5 & Al-SBA-15 \\
\hline \multicolumn{2}{|c|}{ Conversion (wt. \%) } & 26.4 & 70.6 & 91.5 & 58.3 \\
\hline \multirow{10}{*}{$\begin{array}{l}\text { Product yields } \\
\text { (wt. \%) }\end{array}$} & Total gas & 7.9 & 30.7 & 48.8 & 17.0 \\
\hline & Dry gas & 1.6 & 2.6 & 8.2 & 2.2 \\
\hline & LPG & 2.1 & 22.1 & 33.7 & 8.6 \\
\hline & $\mathrm{C}_{2}-\mathrm{C}_{4}$ olefins & 2.3 & 22.7 & 30.4 & 8.5 \\
\hline & $\mathrm{CO} / \mathrm{CO}_{2}$ & 4.2 & 5.9 & 6.9 & 6.3 \\
\hline & $\mathrm{C}_{5+}$ Gasoline & 17.3 & 32.2 & 35.0 & 34.3 \\
\hline & LCO & 29.0 & 17.7 & 4.0 & 30.4 \\
\hline & $\mathrm{HCO}$ & 44.6 & 11.7 & 4.5 & 11.2 \\
\hline & Coke & 0.1 & 1.4 & 1.2 & 1.7 \\
\hline & Water & 1.1 & 6.3 & 6.5 & 5.3 \\
\hline Selectivity & $-\mathrm{C}_{4}$ olefin (\%) ${ }^{\mathrm{a}}$ & 67.6 & 93.2 & 73.5 & 83.3 \\
\hline
\end{tabular}

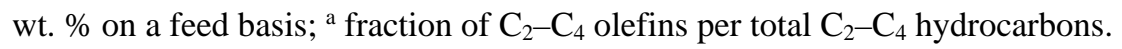

Regarding the product yields, it is obvious that the thermal cracking of WCO gives the lowest yields of desirable products, i.e., gasoline (17.3 wt. \%) and lower olefins (2.3 wt. \%). This is because of lacking acidity; thus, most of the intermediate oxygenated compounds were not converted [6]. The presence of the catalysts generally increases the fractions of gaseous and gasoline hydrocarbons by the cracking of LCO and HCO compounds. However, there are pronounced differences in the product distribution because of the different acid site amount and porosity. With the lowest acid site amount and largest mesopores, Al-SBA-15 produces the most LCO (30.4 wt. \%), but the least $\mathrm{C}_{2}-\mathrm{C}_{4}$ olefins (8.5 wt. \%). In contrast, H-ZSM-5 having the highest acid site amount exhibits the largest fractions of gasoline and 
$\mathrm{C}_{2}-\mathrm{C}_{4}$ olefins (35.0 wt. \% and 30.4 wt. \% respectively). The superior selectivity toward gasoline over $\mathrm{H}-\mathrm{ZSM}-5$ is well documented $[8,16]$. With respect to Al-SBA-15 and H-ZSM-5, the fractions of gasoline and lower olefins obtained with ZSC-24 are ranking in between. However, the outstanding yield for $\mathrm{C}_{2}-\mathrm{C}_{4}$ olefins over H-ZSM-5 mainly results from the high conversion rather than from selectivity since the latter is lowest (73.5 wt. \%) in this series. Remarkably, the highest selectivity to $\mathrm{C}_{2}-\mathrm{C}_{4}$ olefins has been achieved over ZSC-24 (93.2\%), confirming the advantage of ZSC-24 over the reference catalysts. The lower selectivity to $\mathrm{C}_{2}-\mathrm{C}_{4}$ olefins over Al-SBA-15 in comparison with ZSC-24 can be attributed to the shape selectivity of microporous ZSM-5 domains in the latter. However, the low selectivity to $\mathrm{C}_{2}-\mathrm{C}_{4}$ olefins over H-ZSM-5 is probably due to its very high amount of acid sites (mostly located in micropores) and the diffusion constraints imposed by the small pore size of H-ZSM-5 [13]. This might lead to elongated residence times and undesired consecutive reactions of the olefins. Tago et al. [18,19] studied the effect of crystal sizes and acidic properties on the catalytic performance of H-ZSM-5 in the cracking of nhexane at high temperature $\left(550-650^{\circ} \mathrm{C}\right)$. Reducing the crystal size to nano-scale led to rapid diffusion of light olefins out of the intercrystalline micropores of nanosized zeolites as result of the shortened diffusion path lengths, which helped avoid the further transformation of these olefins. This clearly indicates the detrimental effect of internal mass transfer limitation in H-ZSM-5. On the other hand, increasing the number of acid sites enhanced the conversion of n-hexane but decreased the selectivity to light olefins. They postulated that the higher amount of acid sites promoted hydrogen transfer reactions which consumed light olefins to produce gasoline aromatics and light paraffins. A similar behavior can be seen from Figure 2 wherein H-ZSM-5 displays higher concentrations of light paraffins and aromatic hydrocarbons than ZSC-24, confirming the acceleration of hydrogen transfer reactions over H-ZSM-5 due to its higher acid site amount. Thus, the remarkable selectivity to $\mathrm{C}_{2}-\mathrm{C}_{4}$ olefins over the ZSC-24 catalyst can be attributed to the improved molecular transport being well balanced with the medium acid site amount which enables ZSC-24 not only to promote the formation of light olefins, but also effectively to protect them from further transformation.

To discuss the effect of feedstock composition, the results obtained from the cracking of triolein and WCO under the same conditions (ZSC-24, $550^{\circ} \mathrm{C}$ and CTO ratio $=0.4 \mathrm{~g} \cdot \mathrm{g}^{-1}$ ) are used (Tables 3 and 4). It appears that the cracking of WCO produces more light olefins but less gasoline than the cracking of triolein. However, these observed differences in the yields of gasoline and lower olefins are not significant compared to that reported by Dupain et al. [6], who converted triglyceride-based feedstock with FCC catalysts under realistic FCC conditions $\left(525^{\circ} \mathrm{C}\right.$ and $4 \mathrm{~s}$ ). They found that the cracking of saturated stearic acid produced considerably higher yields of gasoline and light olefins (approximately 57 and 14 wt. \%, respectively) than the cracking of unsaturated rapeseed oil (approximately 34 and $3 \mathrm{wt}$. \% for gasoline and light olefins, respectively). It was postulated that the fast aromatization of unsaturated fatty acid over the large pore zeolite Y-based catalyst led to the formation of polyaromatics that are recalcitrant against further cracking. Taking this into account, the minor shift in the yields of gasoline and lower olefins over ZSC-24 confirms that the formation of polyaromatics has been largely suppressed. 

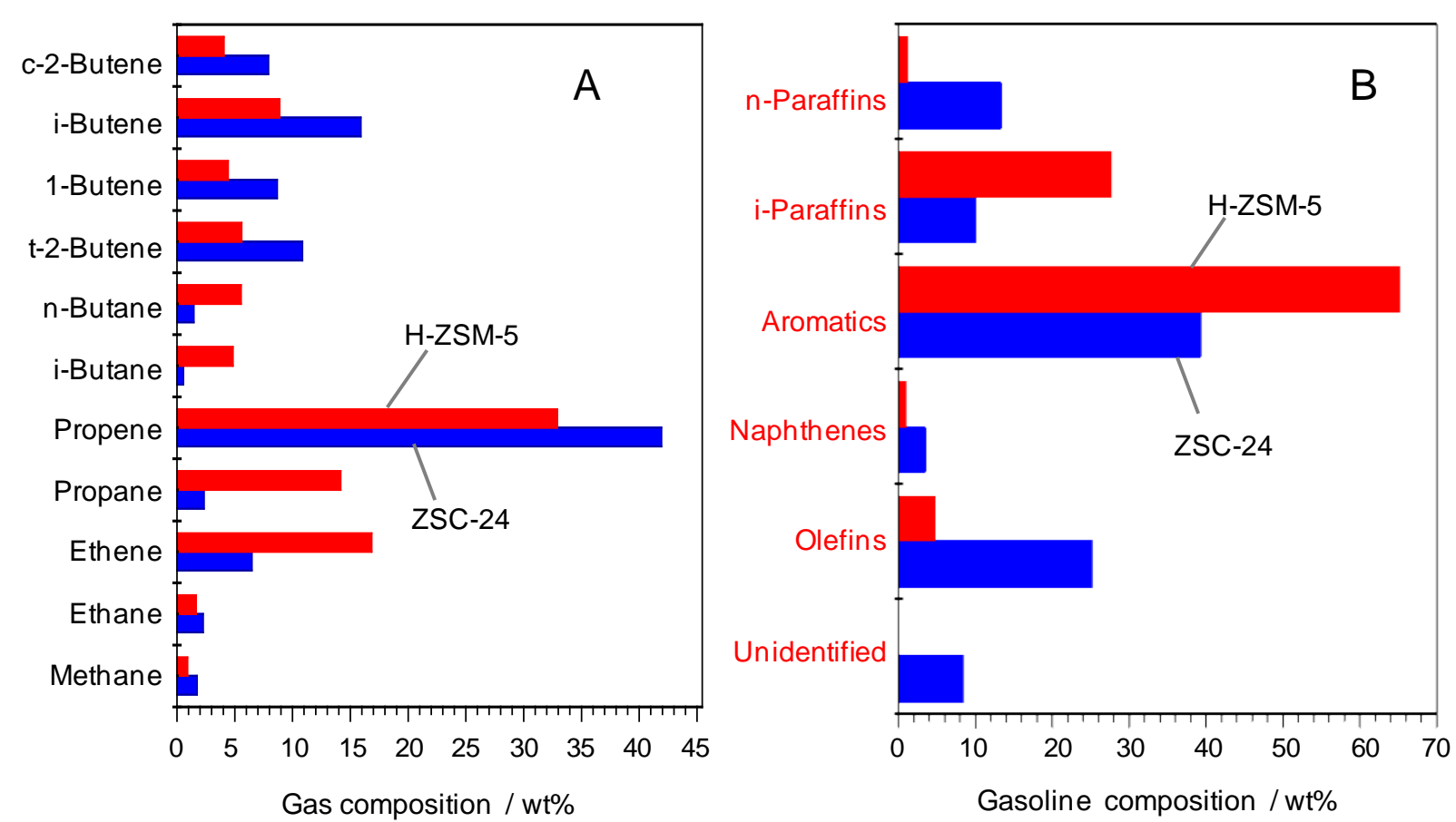

Figure 2. Gaseous hydrocarbon (A) and gasoline (B) compositions in the catalytic cracking of WCO over ZSC-24 and H-ZSM-5 at $550{ }^{\circ} \mathrm{C}$ and a CTO ratio of $0.4\left(\mathrm{~g} \cdot \mathrm{g}^{-1}\right.$ ) (wt. \% on a product basis).

\section{Experimental Section}

\subsection{Catalyst Preparation}

The representative nano-ZSM-5/SBA-15 analog composite, pre-crystallized for $24 \mathrm{~h}$ (denoted as ZSC-24), was prepared according to the synthesis procedure as reported in the previous work [14]. Briefly, the zeolite seed solution was pre-crystallized for $24 \mathrm{~h}$ to partially form nano-ZSM- 5 crystals and then added to the surfactant solution to convert unreacted precursors into an ordered mesoporous SBA-15 analog. Finally, the resulting nano-ZSM-5/SBA-15 analog composite (ZSC-24) was activated by protonation. For comparison, Al-SBA-15 was prepared under the same conditions as for synthesis of ZSC-24, but using conventional silica-based precursors instead of pre-formed seeds. Additionally, commercial H-ZSM-5 (Zeocat PZ-2/25, ZeoChem AG, Uetikon am See, Switzerland) was used to provide a benchmark. All catalyst samples used in this study were fresh since they provide a better understanding of structure, property, and function relationships.

\subsection{Catalyst and Feedstock Characterization}

The nitrogen physisorption studies were carried out at $-196{ }^{\circ} \mathrm{C}$ with an ASAP 2010 apparatus (Micromeritics GmbH, Aachen, Germany). The temperature-programmed desorption of ammonia (NH3-TPD) measurements using TCD detector (TCD, GOW-MAC Instrument Co., Bethlehem, PA, USA) were performed in a homemade set-up using a quartz tube reactor in the range of $100-550{ }^{\circ} \mathrm{C}$. The $\mathrm{Al}$ and Si contents were determined by inductively coupled plasma atomic emission spectroscopy (ICP-AES; 715-ES, Varian, Inc., Palo Alto, PA, USA) and atomic absorption spectroscopy 
(AAS; Analyst 300, PerkinElmer, Inc., Waltham, MA, USA), respectively. More details of these characterization methods and experiment parameters are described elsewhere [14,20].

The fatty acid compositions of technical grade triolein (obtained from Santa Cruz Biotechnology, Inc, Heidelberg, Germany) and waste cooking oil (collected from a local restaurant) were analyzed on a gas chromatography-mass spectrometry combination (GC-MS; QP2010S, Shimadzu Europa GmbH, Duisburg, Germany). For this purpose, a derivatization was carried out with trimethylsulfonium hydroxide solution (0.25 M in methanol, Fluka, Sigma-Aldrich Chemie GmbH, Taufkirchen, Germany) to transform triglycerides completely into methyl esters. The $\mathrm{C}, \mathrm{H}, \mathrm{N}$ elemental analysis of triolein and WCO was performed on a TruSpec CHNS Micro analyser (Leco Instrumente GmbH, Mönchengladbach, Germany).

\subsection{Catalyst Tests}

The fixed bed microactivity test equipment (MAT) is widely used for studying catalytic cracking at the laboratory scale. However, the traditional MAT unit provides cracking results of limited precision and accuracy due to the problems related to the fluid dynamics differences between a fixed bed and a fluidized bed, the large contact time (longer than industrial FCC operations, typically about 2 to 10s), low temperature in the preheating section, or low pressure in the catalyst bed. Most of these shortcomings encountered with the traditional MAT unit can be avoided by using a novel Single Receiver Short-Contact-Time Microactivity Test unit (SR-SCT-MAT, Grace GmbH \& Co.KG, Frankfurt, Germany) [15]. Details of the experimental setup of the SR-SCT-MAT unit, the testing conditions, and product analyses used in the present work are described elsewhere [13].

In a typical run, $1.75 \mathrm{~g}$ of feedstock (either triolein or WCO) was fed into the reactor which contained a desired amount of catalyst diluted with glass beads to maintain a constant-volume reaction independent of the catalyst-to-oil (CTO) mass ratio used. The CTO mass ratio was varied by keeping the weight of feedstock constant (1.75 g) and changing the catalyst weight. The cracking reaction was carried out at ambient pressure, $550^{\circ} \mathrm{C}$, CTO mass ratios of $0-1.2\left(\mathrm{~g} \cdot \mathrm{g}^{-1}\right)$ and a reaction time of $12 \mathrm{~s}$. After the reaction, stripping of the catalyst was done by using a nitrogen purge. The gaseous and liquid products were collected in the single receiver cooled to $18{ }^{\circ} \mathrm{C}$ via an external cooling system. All catalytic experiments were repeated at least two times to check the reproducibility and mass balances in all runs were between $95 \%$ and $100 \%$ of the injected feed.

The products comprised mainly hydrocarbons along with oxygenated compounds (water, CO, and $\mathrm{CO}_{2}$ ) and coke. The gaseous hydrocarbon fraction was divided into dry gas (hydrogen, methane, ethane, and ethene) and liquefied petroleum gas (LPG; propane, propene, butenes, and butanes). The liquid hydrocarbons were lumped in terms of boiling ranges: $\mathrm{C}_{5+}$ gasoline $\left(<221{ }^{\circ} \mathrm{C}\right)$, light cycle oil (LCO; 221-360 ${ }^{\circ} \mathrm{C}$ ), and heavy cycle oil (HCO; $>360^{\circ} \mathrm{C}$ ).

The gaseous products were analyzed according to the ASTM D1945-3 method using a Refinery Gas Analyzer (Agilent 7890A, Santa Clara, CA, USA). The liquid organic products were classified according to the boiling ranges: $\mathrm{C}_{5+}$ gasoline, LCO, and HCO as mentioned above by means of simulated distillation (ASTM D2887) on a Simulated Distillation gas chromatograph (Agilent 7890A, Santa Clara, CA, USA). For several liquid samples, PIONA (Paraffin, i-Paraffin, Olefin, Naphthene, and Aromatic) analyses were performed to determine the composition of gasoline. Water content was measured by Karl 
Fischer titration (MKS-520, Kem, Kyoto, Japan) and coke amount on the spent catalyst was determined by an elemental analyzer (CS600, Leco Instrumente GmbH, Mönchengladbach, Germany).

The yield toward different products ( $\mathrm{Y}_{\mathrm{i}}$, wt. \%) is defined as gram of product i per gram of the feed. The standard MAT conversion is defined as $100 \%-\left(\mathrm{Y}_{\mathrm{HCO}}+\mathrm{Y}_{\mathrm{LCO}}\right)$. The selectivity to $\mathrm{C}_{2}-\mathrm{C}_{4}$ olefins is defined as the fraction of $\mathrm{C}_{2}-\mathrm{C}_{4}$ olefins per total fraction of $\mathrm{C}_{2}-\mathrm{C}_{4}$ hydrocarbons.

\section{Conclusions}

We have shown that ZSC-24 is an efficient catalyst for the cracking of triglyceride-rich biomass toward lower olefins under FCC conditions. Compared to Al-SBA-15 and commercial H-ZSM-5, the ZSC-24 catalyst exhibits the highest selectivity to $\mathrm{C}_{2}-\mathrm{C}_{4}$ olefins (>90\%) irrespective of the cracking severity and feedstock composition. The effect of the catalyst's porosity and acid site amount on the conversion and product distribution was found to be significant. Medium acid site amount, shape selectivity, and improved molecular transport obtained by the high dispersion of nano-scaled ZSM-5 domains in a mesoporous SBA-15 analog matrix might be responsible for the enhanced formation of desired lower olefins. These findings might open the way for future research in the rational design of new FCC catalysts for efficient conversion of triglyceride-rich biomass toward lower olefins. However, it should be noted that the fresh catalysts used in this study might not provide the same results as if the catalysts had been aged.

\section{Acknowledgments}

Financial support from VIED and LIKAT is gratefully acknowledged.

\section{Author Contributions}

X.H.V., T.T.D. and U. A. designed the experiments. T.T.D., X.H.V. and S.N. conducted the experiments. B.M.Q.P., D.A.N., U.A. and A. M. analyzed the data. X.H.V. wrote the first draft of the manuscript which was then revised by all other authors.

\section{Conflicts of Interest}

The authors declare no conflict of interest.

\section{References}

1. Ren, T.; Patel, M.; Blok, K. Olefins from conventional and heavy feedstocks: Energy use in steam cracking and alternative processes. Energy 2006, 31, 425-451.

2. Rahimi, N.; Karimzadeh, R. Catalytic cracking of hydrocarbons over modified ZSM-5 zeolites to produce light olefins: A review. Appl. Catal. A 2011, 398, 1-17.

3. Maher, K.D.; Bressler, D.C. Pyrolysis of triglyceride materials for the production of renewable fuels and chemicals. Biores. Technol. 2007, 98, 2351-2368.

4. Melero, J.A.; Iglesias, J.; Garcia, A. Biomass as renewable feedstock in standard refinery units. Feasibility, opportunities and challenges. Energy Environ. Sci. 2012, 5, 7393-7420. 
5. Huber, G.W.; Corma, A. Synergies between bio- and oil refineries for the production of fuels from biomass. Angew. Chem. Int. Ed. 2007, 46, 7184-7201.

6. Dupain, X.; Costa, D.J.; Schaverien, C.J.; Makkee, M.; Moulijn, J.A. Cracking of a rapeseed vegetable oil under realistic FCC conditions. Appl. Catal. B 2007, 72, 44-61.

7. Rao, T.V.M.; Dupain, X.; Makkee, M. Fluid catalytic cracking: Processing opportunities for Fischer-Tropsch waxes and vegetable oils to produce transportation fuels and light olefins. Micropor. Mesopor. Mater. 2012, 164, 148-163.

8. Chen, D.; Tracy, N.I.; Crunkleton, D.W.; Price, G.L. Comparison of canola oil conversion over MFI, BEA, and FAU. Appl. Catal. A 2010, 384, 206-212.

9. Idem, R.O.; Katikaneni, S.P.R.; Bakhshi, N.N. Catalytic conversion of canola oil to fuels and chemicals: Roles of catalyst acidity, basicity and shape selectivity on product distribution. Fuel Process. Technol. 1997, 51, 101-125.

10. Katikaneni, S.P.R.; Adjaye, J.D.; Bakhshi, N.N. Studies on the catalytic conversion of canola oil to hydrocarbons: influence of hybrid catalysts and steam. Energy Fuels 1995, 9, 599-609.

11. Katikaneni, S.P.R.; Adjaye, J.D.; Idem, R.O.; Bakhshi, N.N. Catalytic conversion of canola oil over potassium-impregnated HZSM-5 catalysts: $\mathrm{C}_{2}-\mathrm{C}_{4}$ olefin production and model reaction studies. Ind. Eng. Chem. Res. 1996, 35, 3332-3346.

12. Botas, J.A.; Serrano, D.P.; García, A.; Ramos, R. Catalytic conversion of rapeseed oil for the production of raw chemicals, fuels and carbon nanotubes over Ni-modified nanocrystalline and hierarchical ZSM-5. Appl. Catal. B 2014, 145, 205-215.

13. Vu, X.H.; Schneider, M.; Bentrup, U.; Dang, T.T.; Phan, B.M.Q.; Nguyen, D.A.; Armbruster, U.; Martin, A. Hierarchical ZSM-5 materials for an enhanced formation of gasoline-range hydrocarbons and light olefins in catalytic cracking of triglyceride-rich biomass. Ind. Eng. Chem. Res. 2015, 54, 1773-1782.

14. Vu, X.H.; Bentrup, U.; Hunger, M.; Kraehnert, R.; Armbruster, U.; Martin, A. Direct synthesis of nanosized-ZSM-5/SBA-15 analog composites from preformed ZSM-5 precursors for improved catalytic performance as cracking catalyst. J. Mater. Sci. 2014, 49, 5676-5689.

15. Wallenstein, D.; Seese, M.; Zhao X. A novel selectivity test for the evaluation of FCC catalysts. Appl. Catal. A 2002, 231, 227-242

16. Twaiq, F.A.; Zabidi, N.A.M.; Bhatia, S. Catalytic conversion of palm oil to hydrocarbons: performance of various zeolite catalysts. Ind. Eng. Chem. Res. 1999, 38, 3230-3237.

17. Melero, J.A.; Clavero, M.M.; Calleja, G.; Garcia, A.; Miravalles, R.; Galindo, T. Production of biofuels via the catalytic cracking of mixtures of crude vegetable oils and nonedible animal fats with vacuum gas oil. Energy Fuels 2010, 24, 707-717.

18. Tago, T.; Konno, H.; Nakasaka, Y.; Masuda, T. Size-controlled synthesis of nano-zeolites and their application to light olefin synthesis. Catal. Surv. Asia 2012, 16, 148-163.

19. Konno, H.; Tago, T.; Nakasaka, Y.; Ohnaka, R.; Nishimura, J.; Masuda, T. Effectiveness of nano-scale ZSM-5 zeolite and its deactivation mechanism on catalytic cracking of representative hydrocarbons of naphtha. Micropor. Mesopor. Mater. 2013, 175, $25-33$. 
20. Vu, X.H.; Eckelt, R.; Armbruster, U.; Martin, A. High-temperature synthesis of ordered mesoporous aluminosilicates from ZSM-5 nanoseeds with improved acidic properties. Nanomaterials 2014, 4, 712-725.

(C) 2015 by the authors; licensee MDPI, Basel, Switzerland. This article is an open access article distributed under the terms and conditions of the Creative Commons Attribution license (http://creativecommons.org/licenses/by/4.0/). 